EOMmUn Communication et organisation

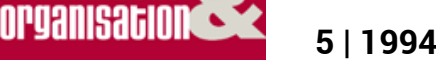

La communication interne : une approche croisée

\title{
L'audit de communication : analyse critique
}

Gilles Willett

\section{OpenEdition}

Journals

Édition électronique

URL : http://journals.openedition.org/communicationorganisation/1699

DOI : 10.4000/communicationorganisation. 1699

ISSN : $1775-3546$

Éditeur

Presses universitaires de Bordeaux

Édition imprimée

Date de publication : 1 mai 1994

ISSN : $1168-5549$

Référence électronique

Gilles Willett, "L'audit de communication : analyse critique », Communication et organisation [En ligne],

5 | 1994, mis en ligne le 26 mars 2012, consulté le 21 décembre 2020. URL : http://

journals.openedition.org/communicationorganisation/1699; DOI : https://doi.org/10.4000/

communicationorganisation.1699

Ce document a été généré automatiquement le 21 décembre 2020.

(c) Presses universitaires de Bordeaux 


\title{
L'audit de communication: analyse critique
}

\author{
Gilles Willett
}

1 Les pratiques d'audit ne sont pas récentes. Aujourd'hui, plus d'une quarantaine de types d'audits sont réalisés par des praticiens individuels ou regroupés dans des cabinets de conseils en communication ou en organisation. Les pratiques d'audit semblent répondre au besoin de mieux contrôler nos usines, nos entreprises et nos institutions qui deviennent de plus en plus complexes.

2 Les problèmes et les conflits de nos milieux de travail, auxquels s'intéressent les auditeurs, sont en fait l'expression de situations compliquées non pas tant à cause de problèmes d'ordre organisationnel, mais parce que l'être humain a la capacité intrinsèque de communiquer et de concevoir. Dès lors, nous tenons pour acquis la nécessité d'accroître la variété des contrôles exercés sur les comportements de communication. Car c'est bien de cela qu'il s'agit, lorsqu'il est question d'audit de communication.

3 Pour mieux saisir ce qu'est l'audit de communication, il convient d'abord de retracer les origines des pratiques d'audit et d'examiner les caractéristiques majeures de différents types d'audit. Par la suite, nous traiterons des facteurs d'émergence et de structuration des pratiques d'audit de communication, nous explorerons les différentes facettes de la fonction, de la tâche et de la formation de l'auditeur et, enfin, nous discuterons brièvement des métaphores, des dimensions oubliées et des mythes ignorés par les praticiens de l'audit de communication mais qui, néanmoins, influencent ces pratiques et leurs résultats.

\section{L'origine des pratiques d'audit}

4 Le mot audit provient du latin auditus qui signifie « entendu ». C'est vers la première moitié du $\mathrm{XX}^{\mathrm{e}}$ siècle que la langue anglaise s'est approprié ce mot pour désigner les actions de vérification, de révision, de contrôle et d'ajustement de la comptabilité et de la 
gestion financière des entreprises par des auditors. Toutefois, les pratiques d'audit sont millénaires.

Selon Filios (1984), les pratiques d'audit ont près de 5000 ans. Elles ont pris naissance avec l'édification de l'Empire Babylonnien où le secrétaire d'État, responsable des affaires financières, consignait dans les documents comptables de l'époque toutes les transactions commerciales importantes et attestait, par le fait même, de leur véracité. Dans l'ancienne Égypte, des percepteurs de taxes très appréciés des Pharaons, tels Joseph de l'Ancien Testament, veillaient à la perception des taxes imposées par l'État sur toutes les importations et les exportations de céréales. Vers l'an 300 avant Jésus-Christ, les Trésoriers publics athéniens étaient soumis au contrôle des logistae qui avaient le pouvoir de traduire devant les tribunaux les personnes qui violaient les lois fiscales, alors que les éfthini, élus par le peuple, exerçaient leur contrôle sur les logistae et sur les fonctionnaires qui avaient pour tâche d'administrer les finances de l'ancienne Grèce. Dans l'Empire romain, les consuls, les magistrats et les trésoriers publics assumaient le contrôle des finances publiques sous la responsabilité du Fiscus.. Mais malgré l'histoire déjà longue des pratiques d'audit, ce n'est qu'en 1581 que les auditeurs professionnels se regroupèrent au sein du Coliegia dei Raxenati de Venise.

6 Depuis l'Empire Babylonnien, l'audit constitue donc une pratique de contrôle des actes des gestionnaires qui, par délégation de pouvoir, administrent des biens et des ressources financières qui ne leur appartiennent pas. Mais aujourd'hui, l'audit ne porte plus essentiellement sur le contrôle des actes de gestion financière. Les types d'audits se sont tellement diversifiés qu'Alain Mikol (1991) n'hésite pas à qualifier la situation actuelle de « jungle des audits ».

7 Cette diversification a permis aux auditeurs d'élargir leurs types d'interventions dans la sphère de l'économie et des finances et d'appliquer des procédures de vérification et de contrôle à d'autres champs d'activités. " Auditer signifiait autrefois vérifier les comptes d'une entreprise pour en certifier l'image fidèle ; aujourd'hui auditer signifie également étudier une entreprise pour en améliorer les performances. Celui-là s'appelle audit financier et celui-ci audit opérationnel. La mission d'audit peut également être qualifiée par son objet (certification de l'image fidèle, amélioration des performances), par son domaine d'investigation (comptabilité, informatique, social, production,...) ou par ses intervenants (commissaire aux comptes, auditeur externe, auditeur interne) (Mikol, 1991, p. 10).

8 Être auditeur n'est pas peu de chose. L'audit donne de la notoriété à ceux qui le pratiquent car il signifie « rigueur, corps de méthodes, encadrement et formation du jeune auditeur, connaissances techniques précises, normes de travail définies par des organismes de révision comptable reconnus, mais aussi salaires très concurrentiels, possibilité de promotion rapide, échelle humaine, sens des responsabilités, et encore développement des budgets pour ne pas dire sens commercial puisque, comme l'on sait, l'auditeur n'est pas un commerçant » (Mikol, 1991, p. 5).

9 Il n'est pas question ici de traiter des facteurs qui ont provoqué la diversification et la croissance des types de pratiques d'audit. Il apparaît toutefois évident que la complexification des systèmes économiques et de ceux de production et de consommation ainsi que la fragmentation croissante des activités humaines ont suscité l'accroissement des actions de vérification, de révision, de contrôle et d'ajustement. Dans la trame de cette tendance globale, l'importance de plus en plus grande accordée à la 
communication dans les organisations ne pouvait pas ne pas entraîner le développement de pratiques d'audit de communication. Mais avant de traiter de ces pratiques, examinons les principales caractéristiques d'un audit.

\section{Les caractéristiques de l'audit}

10 L'examen de différents types d'audit permet de cerner leurs caractéristiques majeures. La signification du mot audit est souvent tenue pour acquise. Mais il y a consensus à l'effet qu'un audit constitue une enquête qui doit être menée de manière circonspecte pour mettre en lumière des problèmes d'écarts par rapport à une norme et pour proposer les manières de les résoudre.

11 Il faut donc distinguer entre audit, diagnostic et analyse et ne pas considérer ces termes comme étant équivalents. L'audit consiste essentiellement à confronter une situation réelle à une situation de référence, alors que le diagnostic sert soit à bien poser le problème soit à rechercher les causes profondes des symptômes caractérisant une situation insatisfaisante. Quant à l'analyse, elle permet de mettre en évidence les éléments constitutifs d'une situation ou d'un problème, ou encore de procéder à une recherche créatrice de solutions dont l'application doit tenir compte de critères financiers, techniques et humains.

12 La réalisation d'un audit est habituellement justifiée par les motifs suivants : Le milieu industriel est un milieu à problèmes ; le personnel est à la fois source de tracas et créateur de richesse ; les nombreux changements actuels nécessitent la compréhension des enjeux pour mieux s'y adapter; il faut que les milieux de travail deviennent des lieux plus humains ; il faut que les administrateurs connaissent plus clairement la manière dont ils sont perçus et qu'ils comprennent l'influence de leurs décisions et de leurs actions; les changements majeurs dans une entreprise sont souvent la source de problèmes et de conflits; il est devenu indispensable de définir des projets d'entreprise ainsi que des politiques et des objectifs; il faut trouver les moyens de créer des consensus ; il faut tenir compte de l'image corporative et des besoins de l'environnement social, etc.. Plusieurs de ces motifs s'apparentent aux mythes d'entreprise et de communication dont il sera question plus loin.

13 L'audit a habituellement pour objectifs de rendre plus efficace et performant, d'améliorer les conditions de réussite, de mieux gérer, de mieux contrôler, de mieux maîtriser une situation problématique, de mieux préparer les décisions ou encore de recentrer les objectifs préalablement déterminés. Tous les types d'audit ont pour but de mettre au service de la direction des moyens de cueillette d'informations, d'examen, d'analyse, de compréhension et de définition de l'état d'une situation, d'aide à la décision, de prévision stratégique et de gestion du changement. L'accent est mis sur l'approche méthodologique opérationnelle et pratique, sur des outils méthodologiques éprouvés et très diversifiés, et sur une démarche systématique selon un nombre variable d'étapes. L'analyse systémique est rarement utilisée. Certains audits sont appliqués à toute l'entreprise, d'autres sont centrés sur les individus et d'autres sur les groupes. Quant aux résultats de l'audit, ils doivent permettre d'établir un plan d'actions, de définir des objectifs et de choisir les moyens appropriés pour les réaliser.

14 L'audit est une pratique d'analyse comparative permettant d'apprécier des écarts par rapport à une norme (politique, loi, règle, coutume etc.), un système ou une situation de 
référence, théorique ou réelle, afin d'en identifier les causes. Il est aussi inductif car l'auditeur doit tenir compte des faits pour remonter à leurs causes et reconstruire les principaux éléments de la situation problématique, son fonctionnement et ses effets pour être en mesure de proposer des solutions réalistes.

Le processus d'audit comporte trois phases : L'étude préliminaire, le travail sur le terrain, puis le rapport. Certains praticiens ajoutent une quatrième phase, celle du suivi de l'audit dont ils prennent la responsabilité si celle-ci ne peut être déléguée à un gestionnaire de l'entreprise auditée.

L'étude préliminaire consiste à clarifier l'intention du client, à rechercher un consensus sur la définition du problème et des mots utilisés pour le représenter, sur les objectifs de l'audit et sur ce qui doit être audité, à faire une analyse des risques de l'audit, à planifier celui-ci puis à définir les étapes de sa réalisation. Si les normes ou les systèmes de référence n'existent pas, il faut les élaborer préalablement à la réalisation de l'audit et obtenir le consensus du client à ce propos. Cette étape vise donc à faire une description détaillée des tâches afin de préciser les éléments du contrat à signer avec l'entreprise. Il s'agit en fait de la préparation du cahier des charges.

17 Le travail sur le terrain est déterminé par les éléments du contrat. Il consiste à réaliser le programme d'audit prévu et à déterminer les matériaux du rapport final. Il doit donc y avoir cueillette et analyse de données et d'informations préliminaires (documents sur l'histoire et le fonctionnement de l'entreprise etc.) sur lesquels sera fondée la stratégie de l'audit. Les outils de cueillette de données sont nombreux et tirés des méthodes traditionnelles d'analyse des sciences humaines (analyse documentaire, questionnaires, entrevues, journal de bord, auto-rapport, observation directe et indirecte, évaluation des attitudes, de la technologie, analyse de perception, de contenu, de tâches, de besoins, de performance, groupes de discussion, construction de typologie, etc.). La cueillette de données doit suivre un cheminement logique qui guide l'auditeur, à chacune des étapes de l'audit, vers la rédaction de son rapport et l'aide à structurer sa pensée et son discours.

Le rapport est constitué, d'une part, de l'analyse et de l'interprétation des données recueillies et, d'autre part, des recommandations. Le contenu du rapport doit faire un rappel du problème posé, des limites de l'audit et des enjeux suscités par celui-ci. Il doit y être question de la nature et du déroulement de l'audit et l'auditeur doit énoncer les commentaires qui facilitent la compréhension de la situation. Les recommandations doivent être réalistes, réalisables et structurées de manière à tenir compte du contexte, des différents aspects de la situation à corriger et des coûts de réalisation.

Les différentes caractéristiques dont il vient d'être question permettent de mieux cerner où en est l'état de l'art des pratiques d'audit de communication. Elles permettent aussi de constater que les praticiens de l'audit, tout en voulant faire pragmatique, négligent de tenir compte des théories et des modèles tant ordinaires que scientifiques qui, pourtant, conditionnent les actions de communiquer et d'organiser dans les différents milieux de travail. Il est d'ailleurs très rare qu'un praticien accepte de livrer sa théorie.

\section{L'audit de communication}

20 Les pratiques d'audit de communication n'ont pas encore un demi-siècle. Elles se situent dans le prolongement des théories et des études sur l'organisation et sur la communication dans les organisations. Depuis toujours, les analystes de l'organisation et 
ceux de la communication dite organisationnelle font appel à plusieurs disciplines des sciences humaines et des sciences sociales, et s'affrontent sur le même terrain. Aujourd'hui, les premiers sont de plus en plus obligés, par les faits, de tenir compte de la communication, alors que les seconds sont de plus en plus conscients qu'ils ne peuvent négliger les impératifs de l'organisation.

21 Le premier audit de communication est attribué à Jacobson et Seashore (1951). Ces chercheurs ont analysé les interactions interpersonnelles de travail dans une agence gouvernementale américaine. Ils avaient pour buts, d'une part, de décrire cette organisation dans une perspective communicationnelle afin d'établir les liens entre ce qui est d'ordre communicationnel et d'ordre organisationnel et, d'autre part, de comprendre l'importance de la communication dans les relations entre le fonctionnement et l'efficacité des entreprises. Ils ont donc recherché les liens entre la productivité, la satisfaction des travailleurs et les différentes manières d'établir les relations de rôles hiérarchiques. Ils ont aussi étudié les différences entre les relations immédiates dans les unités de travail, celles entre des personnes de même statut ainsi que celles entre des personnes de rôles complémentaires.

22 Jacobson et Seashore postulent que certaines organisations permettent aux individus et aux groupes de réaliser leurs propres buts alors que d'autres empêchent les individus de tirer satisfaction du fait d'être intégré dans une structure d'activités ce qui, par conséquent, freine ou même bloque leur collaboration à la réalisation des objectifs de l'organisation. De plus, ils supposent que certaines fonctions techniques ou professionnelles sont génératrices de besoins (réputation, productivité, etc.) qui suscitent des attitudes et des comportements spécifiques. Ils considèrent aussi que les attitudes sont fondées sur l'interprétation de situations relativement ambiguës et que plusieurs des signaux nécessaires à l'interprétation de ces situations sont transmis par l'intermédiaire de communications lors de relations interpersonnelles.

23 Les structures et les fonctions organisationnelles, le contenu des échanges, les supports et les canaux de communication, le climat des relations interpersonnelles, les styles de communication et leurs effets psychologiques sont les principaux aspects examinés par ces chercheurs Leur étude démontre qu'il est possible de concevoir et de conceptualiser, d'une manière communicationnelle, une structure organisationnelle lorsque l'analyse est fondée sur les événements de communication qui définissent les modèles d'interactions et de relations entre des individus et entre des groupes. Lorsque ces événements de communication sont répétitifs, ils acquièrent des caractéristiques particulières de structure, de fonction, de contenu ainsi que d'effets psychologiques, et constituent des données pertinentes et utiles pour l'analyse communicationnelle d'une organisation.

24 Les auteurs notent aussi que certaines personnes ont effectivement plus de pouvoir que ne leur confère leur fonction dans l'organisation, alors que d'autres sont dans la position inverse. De plus, ils considèrent que c'est la communication qui définit les modèles d'interactions et de relations entre les individus et non pas la structure organisationnelle. Enfin, ils précisent que des problèmes méthodologiques énormes doivent être résolus avant qu'il soit possible de rendre compte de la complexité communicationnelle d'une organisation.

25 Davis (1953) tentera de résoudre certains problèmes méthodologiques, identifiés par Jacobson et Seashore, en développant une nouvelle technique de cueillette de données qu'il nomme l'Ecco Analysis (Episodic Communication Channels in Organisation). Cette 
technique, dérivée de l'analyse des réseaux utilisée par Jacobson et Seashore, est constituée d'un questionnaire permettant de recueillir les données nécessaires pour suivre le cheminement d'informations dans les réseaux de communications internes d'une organisation.

Lorsque Odiorne (1954) publie les résultats de son étude exploratoire sur une entreprise de recherche et développement en défense militaire, il utilise pour la première fois l'expression "audit de communication ». Cette entreprise est confrontée à des conflits issus de problèmes de croissance, de recrutement et de roulement du personnel, d'absence de compréhension réciproque et de coopération entre les employés ainsi que de relations humaines impersonnelles entre les contremaîtres et les ouvriers. Son instrument de cueillette de données est constitué d'un questionnaire de la National Society of Professional Engineers (NSPE, 1952) qu'il adapte aux besoins de son analyse.

Odiorne cherche à comprendre le flux et l'exactitude des communications dans cette entreprise et à évaluer l'efficacité de la transmission des informations, plutôt que la qualité de ce qui est transmis. Son audit porte sur les aspects suivants : l'opinion des ingénieurs sur la pertinence des canaux de communication, sur l'exactitude et la complétude des informations transmises dans l'entreprise ainsi que la perception des ouvriers quant aux canaux de communication, à la circulation descendante et ascendante des informations et à leur degré d'acceptation de celles-ci.

En conclusion de son étude, Odiorne fait ressortir les limites de celle-ci et observe que les techniques d'audit restent à être validées. De plus, il précise qu'il faudrait mieux comprendre la nature de la communication dans une organisation pour être en mesure d'en traiter correctement. Il faut attendre le début des années 1970 avant que l'International Communication Association (ICA) tente de résoudre, avec les travaux de Goldhaber $(1974,1977,1978 \mathrm{a}, 1978 b, 1984)$ et son équipe, les problèmes complexes que pose la réalisation d'un audit de communication.

\section{Structuration de l'audit de communication}

L'ICA Communication Audit est né du constat de plusieurs lacunes. La plupart des recherches sur la communication dans les organisations n'utilisaient qu'un seul instrument de cueillette de données. Les résultats n'étaient pas généralisables à d'autres entreprises. L'échantillonnage des personnes interviewées était petit et composé presque essentiellement de gestionnaires et de professionnels. La procédure d'audit et les instruments de mesure n'étant pas standardisés, il était impossible de faire l'analyse comparative des comportements de communication et de mesurer l'écart par rapport à une norme. De plus, les études pouvaient difficilement être validées, les mesures et l'évaluation des attitudes et des perceptions des comportements de communication étaient grossières et limitées dans le temps et, enfin, les instruments de mesure utilisées ne permettaient pas d'énoncer des prédictions valables parce qu'il y avait très peu de données sur la performance des organisations.

Pour combler ces lacunes, les chercheurs de l'ICA ont défini une procédure systématique d'audit et des normes quant aux instruments de cueillette de données et au contenu du rapport final. Ils ont aussi constitué une banque de données sur les systèmes de communication des entreprises étudiées afin de rendre possibles les analyses 
comparatives nécessaires à la validation de la méthodologie de l'audit et de plusieurs théories relatives à la communication organisationnelle.

31 La réalisation de l'audit de communication de l'ICA est fondée sur les huit objectifs suivants: Déterminer la quantité d'informations par rapport aux sources, aux sujets traités et aux canaux de communication ; évaluer la qualité des messages transmis par ou vers différentes sources d'informations; estimer la qualité des relations communicationnelles en mesurant le degré d'importance de la confiance interpersonnelle ainsi que le degré de support mutuel, de sociabilité et de satisfaction tirée de la tâche accomplie; identifier les réseaux informels de communication et les comparer aux réseaux formels; identifier les blocages qui se manifestent dans la circulation de l'information ainsi que les modes de sélection des messages en comparant les comportements réels de communication des individus à ceux définis par la description de la tâche à accomplir; décrire les comportements de communication des individus et des groupes par rapport aux sources d'informations, aux canaux de transmission des informations, au sujet des échanges et par rapport à la durée et à la qualité des interactions; énoncer des recommandations permettant de modifier les attitudes, les comportements, les pratiques et les habiletés de communication.

32 La cueillette de données nécessaires pour atteindre ces objectifs exige l'utilisation de cinq instruments: des entrevues préliminaires et en profondeur; des questionnaires, la description des différentes formes d'expériences de communication dans leur entreprise par les individus audités ; l'analyse des réseaux sociométriques ; la rédaction d'un journal de bord par chaque personne auditée où sont consignées toutes les interactions établies aux cours de durées prédéterminées, ainsi que les facteurs qui ont influencé ces interactions. L'analyse croisée de toutes les données recueillies permet d'établir l'état de la situation et d'identifier les différents problèmes de communication et d'information.

Malgré l'aspect systématique de cette procédure, l'audit de communication de l'ICA comporte plusieurs défauts dont ont traité Sincoff et Goyer (1977), Goldhaber et Krivonos (1977), Brooks, Callicoat et Siegerds (1978) ainsi que DeWine et James (1988). Selon DeWine et James (1988, p. 145-146), l'audit de l'ICA n'a pu établir un contrôle centralisé sur le processus de cueillette des données. Il s'est avéré impossible de comparer les entreprises locales par rapport à une norme nationale. Il n'y a aucune procédure de suivi permettant d'évaluer les conséquences de l'application des recommandations du rapport d'audit. Les instruments de mesure sont limités à l'évaluation des perceptions des individus. Il y a de nombreux problèmes à résoudre quant à la rigueur du processus d'audit, aux méthodes d'analyse des données et à la manière d'énoncer des recommandations fondées sur l'interprétation des résultats.

Toujours selon DeWine et James (1988, p. 158-159), il est impossible de tenir pour acquis que l'audit de l'ICA constitue un moyen permettant de rendre plus efficace une organisation. Les résultats de l'audit ne donnent qu'une image générale du climat de communication de l'organisation, image qui peut concorder ou non avec la perception que les administrateurs ont de la situation. D'ailleurs, ceux-ci acceptent d'abord et avant tout les analyses qui confirment leurs perceptions de la réalité organisationnelle et ce qu'ils croient le plus approprié pour leur entreprise, quelle que soit la nature des recommandations proposées. Néanmoins, l'audit leur permet d'identifier ou de prendre conscience des problèmes de communication et des solutions possibles, de découvrir de nouvelles manières d'agir, de confirmer les actions en cours, de susciter leur créativité pour résoudre les problèmes ou encore « de faire le mort ». De plus, les recommandations 
peuvent constituer un moyen de faire comprendre l'importance des comportements de communication, d'être en mesure de les observer, de les évaluer et de les modifier.

À ces critiques, il faut ajouter que la standardisation de la procédure d'audit de communication par l'ICA a permis la création d'une boîte à outils méthodologiques, mais n'a pas transformé les cadres théoriques sur la communication dans les organisations. C'est comme si la mécanique organisationnelle avait orienté la démarche des chercheurs et avait inhibé leur capacité d'énoncer de nouvelles manières de modéliser la communication dans les organisations. Aujourd'hui, l'audit de l'ICA ne constitue qu'une procédure méthodologique longue, coûteuse et compliquée qui ne permet pas de rendre compte de la nature de la communication dans une organisation ni de sa complexité, tel que le souhaitait Odiorne. Et même si la procédure d'audit de l'ICA semble centrée sur la compréhension d'événements de communications, les nombreux résultats accumulés ne permettent pas de concevoir, d'une manière communicationnelle, une structure organisationnelle comme l'indiquaient Jacobson et Seashore.

Étant donné que les chercheurs ne tiennent pas compte du fait que tout être humain est fondamentalement un être de communication capable intrinsèquement de création de réalités physique et symbolique, il sera difficile de résoudre les problèmes théoriques et méthodologiques de l'audit de communication. De plus, il faudrait que ces mêmes chercheurs prennent conscience du fait que l'action de communiquer et celle d'organiser renvoient à des phénomènes à la fois complémentaires et en opposition. À défaut de cela, il leur sera impossible, de comprendre comment s'articulent ces deux actions: celle de communiquer, avec tout ce que cela comporte d'incertitude et de potentiel de changement, et celle d'organiser avec tout ce que cela implique de certitude et de maintien de ce qui existe, tout au moins à court terme.

Des auteurs tels Greenbaum (1974), Strenski (1978, 1984), Washbrook (1978), Gildea et Rosenberg (1979), Machin et Tai (1979), Hickson (1980), Valentine (1981), Wirtz (1981), Kopec (1982), Booth (1986), Hamilton (1987), Downs (1988), Henriet (1990) et bien d'autres, ont traité de l'audit de communication. Cependant, il revient à Claire Badaracco (1988) de souligner les aspects politiques sous-jacents aux audits de communication.

Badaracco considère que l'audit de communication est l'affaire des praticiens des relations publiques. Elle précise que deux écoles de pensée définissent ainsi les pratiques de ce type d'audit: d'une part, c'est un moyen pour aider les communicateurs à gérer et, d'autre part, c'est un outil pour aider les gestionnaires à communiquer. De plus, elle estime que l'audit de communication est à la fois une technique traditionnelle de recherche dans le monde des affaires et un nouvel outil à connotation politique. Cette connotation vient du fait que les pratiques d'audit de communication mettent en cause ce que sont les relations publiques, en leur ajoutant la crédibilité de la recherche scientifique en sciences humaines et sociales, et font évoluer ces pratiques vers une fonction de gestion de la communication. Ce dernier aspect ne fait cependant pas l'unanimité chez les relationnistes.

39 Un audit de communication comporte toujours, selon Badaracco, une interprétation politique des faits par crainte d'un effet boomerang. Lorsque les nouvelles sont mauvaises, le client a tendance à vouloir «tuer le messager " et à ignorer les solutions proposées. De plus, le relationniste qui se considère comme un auditeur ne devrait pas négliger de réfléchir aux problèmes d'éthique et de conflit d'intérêt sérieux auxquels il devra faire face. Ces problèmes, d'ordre politique, se situent dans un contexte idéologique plus large où la perception américaine du management est enracinée dans le concept de 
l'« élu », lui-même fondé sur l'éthique puritaine. Dans ce contexte, la communication ne peut être conçue comme étant une responsabilité partagée dans l'entreprise et, dès lors, les relationnistes sont peu respectés par les administrateurs de haut niveau.

40 Cette prise de position de Badaracco démontre bien que les pratiques d'audit de communication n'ont pas encore atteint la rigueur des audits financiers. En l'absence d'un cadre théorique qui détermine les balises de l'interprétation des résultats d'un audit de communication, la simple existence d'une boîte à outils méthodologiques ne peut fonder cette rigueur. De plus, cette prise de position pose le problème de la définition de ce qu'est un auditeur.

\section{L'auditeur}

41 Les auteurs dont il a été question jusqu'à maintenant et d'autres tels Vardaman et Vardaman (1974), Ballard (1981), Rigny (1982), Mackenzie (1986) et Harrison (1987) ont traité de la fonction, de la tâche et de la formation de l'auditeur. 11 n'y a pas consensus quant à la manière de nommer les praticiens qui traitent des problèmes de communication dans les organisations. Auditeur, conseiller, animateur, diagnosticien, intervenant, concepteur, formateur sont quelques exemples de la jungle des étiquettes à leur propos. Il existe de nombreuses façons de définir et de percevoir ces praticiens et leurs pratiques, et d'énoncer ce qui est attendu d'eux.

Étant donné que l'auditeur pénètre bien souvent dans le "secret des dieux ", il peut être source d'anxiété, de crainte et de méfiance, même s'il est perçu comme le dernier recours pour dénouer une crise. Solliciter ses services c'est un peu avouer son incompétence ou tout au moins son incapacité à régler un problème. C'est aussi, parfois, rechercher l'expression de critiques par un tiers ou la confirmation de l'existence de problèmes dont la responsabilité est attribuée aux autres, plutôt que la mise en évidence des conflits et de leurs solutions. Si l'auditeur est perçu comme un contrôleur, et pire, comme un intrus qui fait irruption dans la tranquillité agitée des situations conflictuelles, plusieurs individus ont tendance à considérer qu'il ne peut comprendre le fond des problèmes et qu'il n'a pas la compétence pour remettre en cause ce qui, apparemment, fonctionnait bien jusque là.

L'auditeur ne doit pas être trop jeune et il doit avoir de l'empathie pour les autres. Il a de la mémoire, de l'intuition, le sens des responsabilités, une grande honnêteté intellectuelle, un bon jugement et une grande faculté de compréhension. Il est capable d'initiative, de discipline, de fermeté, de discrétion et de sociabilité. Ce doit être une personne dynamique, indépendante d'esprit, ayant une grande maîtrise de soi et le sens de l'organisation et de la décision. Il devrait comprendre et parler plusieurs langues et être au fait des caractéristiques particulières de plusieurs cultures.

Il doit savoir se présenter aux autres et s'adapter à l'esprit local. Il doit avoir accès à la haute direction de l'entreprise auditée et fonder ses relations avec les décideurs sur le respect. Il doit identifier ceux qui détiennent le pouvoir, savoir prendre ses distances par rapport à eux et ne jamais céder aux pressions. Il doit être apte à faire face à une opposition larvée et à traiter de situations complexes et changeantes. Il doit savoir se faire accepter, fonder sa crédibilité et démontrer son professionnalisme et ses aptitudes relationnelles. Il doit éviter les conflits d'intérêt, être objectif, ne pas entrer dans le jeu d'une relation conflictuelle, respecter les intérêts légitimes et la dignité de l'audité, et savoir convaincre. Il doit refuser de juger les personnes et d'enfreindre le code moral en 
vigueur. Il doit éviter de dramatiser les écarts observés ou d'abuser des effets de surprise. Il doit aussi s'astreindre au secret professionnel.

Pour ceux qui, compte tenu des coûts, privilégient une pratique individuelle d'audit, une culture générale et technique de niveau convenable suffirait pour devenir auditeur. Mais il y a consensus à l'effet que la complexité des problèmes à résoudre nécessite la constitution d'équipes multidisciplinaires de recherche.

L'auditeur doit être une personne très informée. Il doit avoir une solide connaissance des sciences du comportement, des méthodes de recherche, de la recherche, des processus organisationnels, du domaine industriel où il exerce sa pratique et de la terminologie spécifique à ce domaine. Il doit pouvoir constituer une équipe multidisciplinaire de recherche et travailler en équipe. Il doit être un psychologue averti, un sociologue, un anthropologue et un ethnologue. Il doit avoir une compréhension approfondie des sciences de l'administration, des relations industrielles et publiques, des principes $\mathrm{du}$ management et du marketing, des systèmes de télécommunication et de traitement de l'information, de l'informatique, de la télématique et de la bureautique. Il doit avoir une expérience pratique de ces domaines et du fonctionnement de l'entreprise auditée.

À la lumière de ces différents aspects, l'auditeur apparaît comme un être mythique. De plus, il faut bien reconnaître que peu de place est faite à la formation en communicologie. Cette situation démontre bien que les pratiques d'audit de communication sont d'abord orientées par une problématique organisationnelle où la communication est soit tenue pour acquise soit considérée comme un aspect, parmi tant d'autres, du fonctionnement d'une entreprise, et non pas comme le moteur de ce fonctionnement.

Dès lors, plusieurs aspects du vécu communicationnel en milieu de travail sont ignorés lors de la réalisation d'un audit de communication. De plus, un nombre important de mythes d'entreprise et de communication orientent soit la réalisation des audits soit l'interprétation de leurs résultats car ces mythes empêchent l'auditeur, d'une part, de tenir compte du fait que tout être humain est et demeure, neurobiologiquement et socialement, un être de communication capable intrinsèquement de création de réalités et, d'autre part, de comprendre comment s'articulent les actions de communiquer et d'organiser.

\section{Les dimensions oubliées et les mythes}

49 Nous ne pouvons pas dans ce court texte faire état des nombreuses conséquences sur les audits de communication qu'entrainent les métaphores et les mythes sur lesquels sont fondées les actions d'organiser et de communiquer. Dès lors, nous nous contenterons de préciser certains aspects que nous considérons comme étant les plus importants. Morgan (1989) a très bien documenté les métaphores qui caractérisent les manières de concevoir l'organisation et le vécu organisationnel. L'organisation vue comme machine, organisme, cerveau, culture, système politique, prison du psychisme, flux et transformation ainsi qu'instrument de domination sont autant de conceptions et de perceptions qui, d'une part, structurent l'articulation des actions de communiquer et d'organiser ainsi que les relations interpersonnelles en milieu de travail et, d'autre part, définissent les comportements de communication tolérés et le bassin des événements de communication possibles. 
51 Les praticiens de l'audit de communication n'ont à peu près jamais accordé d'importance à ces conceptions et à ces perceptions. Pourtant, c'est parce que l'humain est un être de communication qu'il est "un être de parole », « un être spatio-temporel», " un être de désir et de pulsion ", "un être symbolique » et "un être de plaisir et de souffrance » (Chanlat, 1990). L'approche mécaniste et instrumentale des audits de communication ne permet pas de tenir compte de ces dimensions fondamentales du vécu communicationnel de l'être humain.

52 Étant donné que la parole et son exercice sont souvent considérés comme une perte de temps et d'argent par le raisonnement administratif, économique et technique, « communiquer se résume donc à transmettre une information» (Chanlat, 1990, p. 34). Cette perspective réductionniste est souvent privilégiée dans les audits de communication. Serait-ce que les praticiens de l'audit tiennent pour acquis que la pensée, la parole et le langage (mots et gestes) sont la prérogative des administrateurs et des gestionnaires? En concevant de manière aussi étroite l'action de communiquer, il faut s'attendre à ce qu'il y ait inhibition de la créativité individuelle et collective et à ce que la communication ne soit plus qu'un automatisme relationnel ayant pour but de préserver les acquis et la marge de survie psychique de chaque individu.

Cet automatisme relationnel est d'ailleurs en concordance avec l'espace et le temps imposés des milieux de travail. Selon Chanlat (1990, p. 161), «l'espace, lieu à la fois de la survie biologique et de l'existence psychologique, est aussi un lieu social, un champ qui structure les interactions et ce, quels que soient leurs types (local, régional, national, international) ». Quant au temps objectif de l'organisation, il confronte constamment le temps subjectif des individus. L'espace rétréci et le temps compressé des lieux de travail sont devenus des variables quantitatives soumises à des impératifs économiques et techniques d'efficacité, de productivité et de profit qui réduisent, d'une part, les aspects subjectifs et qualitatifs de l'espace et du temps de travail à un indice de satisfaction et, d'autre part, la communication à ce qui est organisationnellement essentiel. Dans la mesure où les audits de communication sont orientés par cette perspective, il devient difficile, sinon impossible, de faire ressortir le fait que l'action de communiquer a son espace et son temps propres et qu'elle est indispensable à la survie des organisations.

Bien que les désirs, les pulsions et l'intériorité de l'humain constituent des aspects fondamentaux de l'action de communiquer et même de celle de gérer, Chanlat estime que ces aspects sont oubliés, occultés et même niés par l'organisation actuelle du travail. Pourtant, l'observation permet facilement de constater que le plaisir et la souffrance dans le travail sont l'expression d'une confrontation entre une organisation du travail déficiente et la vie psychique des travailleurs. Pour résoudre cette confrontation, la motivation n'est qu'une manière de « remplacer le vide symbolique créé par la division du travail et la fragmentation croissante de l'existence contemporaine " (Chanlat 1990, p. 261). À l'évidence, l'audit de communication, tel qu'il est pratiqué aujourd'hui, ne peut tenir compte adéquatement de ce qui fonde la dynamique des relations et des rapports interpersonnels supérieur-subordonné, rapports qui sont constamment confrontés au problème de l'harmonisation entre l'action de communiquer et celle d'organiser, actions dont la charge symbolique peut même bloquer toutes possibilités d'interactions.

Comme être de communication, l'humain est un « fabriquant de sens et un producteur de symboles » et l'organisation « en tant qu'univers singulier, produit des phénomènes qui relèvent de l'ordre du symbolique »(Chanlat 1990, p. 531). Or, le signe organisationnel, qui ne fait qu'informer, appauvrit l'organisation symbolique du travail, génère du sens 
qui peut menacé la survie de l'organisation et provoque l'absence des symbolisations unifiantes qui permettent de contrer le climat déstructurant du "travail en miette" (Friedmann, 1964) et de la fragmentation des esprits. L'audit de communication tient peu compte de ces aspects alors même que le symbolique et la signification sont au cœur de l'action de communiquer et du sentiment d'appartenance à une organisation.

Aux dimensions oubliées dont traite Chanlat, il faut ajouter les mythes qui conditionnent les comportements organisationnels et communicationnels ainsi que certaines pratiques d'audit. Selon Pémartin (1990), les mythes d'entreprise induisent des comportements de communication qui génèrent des problèmes et des conflits en milieu de travail.

Prenons les exemples suivants. Autrefois, le personnel était plus attaché à son travail ! Les subalternes refusent d'assumer les responsabilités! Les conflits sont l'expression de la mauvaise volonté des salariés! La vitesse actuelle du changement est une aliénation pour l'individu et l'organisation! La réussite du changement dépend seulement de la qualité des décisions prises par les dirigeants! Les décisions des dirigeants sont rationnelles (alors que celles des subalternes sont affectives!) Beaucoup de dysfonctionnements sont dus à un contrôle insuffisant de l'activité des subordonnés! Le cadre efficace se préoccupe en priorité des problèmes de production et d'organisation! Les technologies nouvelles améliorent la circulation de l'information!

Au cœur de chacun de ces mythes, qui paraissent porter essentiellement sur des aspects organisationnels, il y a des dimensions d'ordre communicationnel qui définissent et orientent la qualité des relations humaines en milieu de travail. Bien souvent, ces mythes empêchent de comprendre non seulement la nature de la communication organisationnelle mais aussi le fait que ce qui est au cœur des actions et des activités organisationnelles, c'est l'action de communiquer, c'est la possibilité, pour chaque individu, de créer du sens au delà de la fragmentation nécessaire à l'existence d'un haut degré de certitude organisationnel. Dès lors, la planification, la définition d'objectifs, de buts, de normes et de règles ont pour effet de contraindre les comportements de communication et de constituer un environnement symbolique qui cristallise les fragmentations organisationnelles alimentées par une juxtaposition de connaissances et de compétences hypertrophiées. Quant aux mythes de communication, ils sont aussi nombreux que ceux d'entreprise. Dionne et Ouellet (1989) font état de ceux qui renvoient non seulement aux pratiques de communication mais également aux fondements de plusieurs modèles de communication.

Prenons les exemples suivants. Il n'existe qu'un niveau de langage qui se manifeste par les écrits ou la parole ! La communication se limite à l'information explicite qui circule entre les individus! La signification d'une communication réside dans les propos échangés! Le fait de communiquer ou non repose sur un choix individuel! Le refus de communiquer met fin à la relation et, conséquemment, à la communication! En matière de communication, il faut se parler pour se comprendre! En matière de communication humaine, l'objectivité est possible! Communiquer, c'est échanger de l'information! La communication porte sur des événements fondés objectivement ! Un message clair induit compréhension et soumission!

Ces mythes sont évidemment réducteurs de la complexité qui caractérise l'action de communiquer. Ils négligent de tenir compte de la communication non-verbale, du nondit, de l'implicite et de ce qui est tenu pour acquis. Ils contribuent à survaloriser l'information transmise et supposément reçue, information qui devient la seule variable d'interprétation d'une situation et du contexte de celle-ci. Ils permettent d'occulter le fait 
que la signification d'un message réside dans les personnes et particulièrement dans la manière dont chaque personne interprète un message. Ils permettent aussi de dissocier l'indissociable, c'est-à-dire la communication et la relation, laissent croire que plus de paroles suscitent plus de compréhension, car la parole et, de ce fait, la communication seraient objectives et, de plus, ils sèment la confusion en établissant une équivalence entre information et communication. Quant à la clarté du message, ce mythe permet d'occulter le fait que tout être humain est libre d'interpréter à sa guise les messages qu'il accepte de recevoir.

61 Les praticiens de l'audit de communication semblent actuellement bien mal outillés pour faire face aux mythes d'entreprise et à ceux de communication, et pour décrypter leurs conséquences sur le vécu communicationnel et sur les difficultés d'harmonisation des actions de communiquer et d'organiser. Cependant, ces mythes suggèrent d'eux-mêmes la nécessité d'une réflexion sur nos manières de concevoir le phénomène de la communication et d'un recadrage de celles-ci.

\section{En guise de conclusion}

62 Hamilton (1987) considère que l'auditeur négocie toujours une nouvelle réalité sociale lorsqu'il intervient dans une situation problématique. C'est ce qui caractérise fondamentalement l'audit de communication et rend ses pratiques très délicates car l'auditeur qui intervient dans un processus de communication est à la fois juge et partie, contrairement à l'auditeur financier.

De plus, ses interventions n'ont pas pour conséquence de traduire quelqu'un devant les tribunaux pour défaut de communication ou d'information. Elles ont pour effets de recadrer la perception de la réalité et de transformer les attitudes et les comportements. Les résultats d'un audit de communication ne peuvent donc être que la somme des conséquences d'actions de vérification, de révision, de contrôle et d'ajustement, ni la simple mesure d'un écart. Si l'audit de communication avait essentiellement pour objet d'accroître le contrôle des décideurs de nos sociétés sur les individus, il faudrait bannir cette pratique qui ne ferait qu'ajouter à la panoplie de ce qui existe déjà dans ce domaine.

Au terme de cette réflexion sur l'audit de communication, il faut bien admettre que cette pratique est orpheline d'une problématique. De plus, en paraphrasant les propos de Katz et Kahn $(1966$, p. 4) sur l'analyse sociologique des structures sociales, il faut se demander si cette pratique ne perd pas de vue l'essentiel, c'est-à-dire le fait que l'être humain est un être de communication? Les praticiens de l'audit de communication se gardent bien de définir ce qu'ils entendent par communication, par information, par donnée, par organisation ou par relation humaine. L'absence d'une problématique ne peut être comblée par une boîte à outils méthodologiques qui, comme par magie, témoignerait à elle seule de la rigueur et de la profondeur de la compréhension d'une situation.

L'audit de communication et ses praticiens ont encore plusieurs défis à relever. Ces derniers devront se constituer en un corps professionnel reconnu. Ils devront fonder leur crédibilité sur l'existence d'une théorie de l'analyse communicationnelle des organisations, sur celle d'une méthodologie éprouvée et sur un code d'éthique reconnu. Ils devront aussi déterminer les sanctions disciplinaires s'appliquant aux cas de manquement aux obligations à la charge de l'auditeur. Enfin, il leur reste à développer des méthodes de travail et de supervision et à définir les règles d'accès à la profession. Ce 
n'est qu'à ces conditions que les auditeurs de communication pourront acquérir de la notoriété et ne pas décevoir les attentes de ceux qui ont recours à leurs services.

\section{BIBLIOGRAPHIE}

Badaracco, C, The Politics of Communication Audits, Public Relations Quarterly, automne 1988, pp. 27-31.

Ballard, W. T., Implementation-Oriented Auditing, The Internal Auditor, février 1981, pp. 15-20.

Booth, A., Communication Audits, a UK Survey. London, Taylor Graham, Primary Communications Research Centre, 1986.

Brooks, K., J. Callicoat et G. Siegerdt, The ICA Communication Audit and Perceived Communication Effectiveness Changes in 16 Audited Organizations, Human Communication Research, vol. 5, $\mathrm{n}^{\circ} 2$, 1979, pp. 130-137.

Chanlat, J. F. (sous la direction de), L'individu dans l'organisation. Les dimensions oubliées. Québec, Les Presses de l'Université Laval/Éditions Eska, 1990.

Davis, K., A Method of Studying Communication Patterns in Organizations, Personnel Psychology, vol. 6, 1953, pp. 301-312.

Dewine, S. et A. C. James, Examining the Communication Audit, Management Communication Quarterly, vol. 2, n 2, 1988, pp. 144-169.

Dionne, P. et G. Ouellet, La communication interpersonnelle et organisationnelle : l'effet Palo Alto. Boucherville, Qc, Gaétan Morin éditeur/Les Éditions d'Organisation, 1990.

Downs, C. W., Communication Audits. Glenview, Ill., Scott, Foresman and Company, 1988.

Filios, V. P., A Concise History of Auditing, The International Auditor, juin 1984, pp. 48-49.

Friedmann, G., Le travail en miette. Paris, Éditions Gallimard, coll. Idées-nrf, 1964.

Gildea, J. A. et K. Rosenberg, Auditing Organizational Communications : Is There Life Beyond Print-Outs ?, University of Michigan Business Review, vol. 31, n 4, 1979, pp. 7-12.

Goldhaber, G. M., Organizational communication. Dubuque, IA, William C. Brown Co., 1974.

Goldhaber, G. M. et P. D. Krivonos, The ICA Communication Audit : Process, Status and Critique, Journal of Business Communication, vol. 15, $\mathrm{n}^{\circ} 1,1977$.

Goldhaber, G. M., O. A. Who, H. S. Dennis III et G. M. Richetto, Information Power and Management Function: Developping Organizational Intelligence. Englewood Cliffs, N.J., Prentice-Hall, 1978a.

Goldhaber, G. M., M. Yates, T. Porter et K. Lesniak, Organizational Communication : 1978, Human Communication Research, vol. 5, $\mathrm{n}^{\circ}$ 1,1978b, pp. 76-96.

Goldhaber, G. M. et D. Rogers, Auditing Organization Communication Systems : The ICA Communication Audit. Dubuque, IA, Kendall/Hunt, 1979.

Goldhaber, G. M, H. S. Dennis III, G. M. Richetto et O. A. Who, Information Strategies. New Pathways to Management Productivity. Norwood, N.J., Ablex Publishing Corporation, 1984. 
Greenbaum, H. H., The Audit in Organizational Communication, Academy of Management Journal, vol. 17, nº 4, décembre 1974, pp. 739-754.

Hamilton, S., A Communication Audit Handbook. London, Pitman Publishing, 1987.

Harrison, M. I., Diagnosing Organization : Methods, Models, and Processes. London, Sage, 1987.

Henriet, B., Audit de la communication interne. Paris, Les Éditions d'Organisation, 1990.

Hickson, M., The Open Systems Model : Auditing the Effectiveness of Organizational Communications, dans P.V. Lewis et J. Williams, Reading in Organizational Communication, Columbus, Ohio, Grid Publishing, 1980, pp. 359-365.

Jacobson, E. et S. E. Seashore, Communication Practices in Complex Organization, Journal of Social Issues, vol. 7, n 3, 1951, pp. 28-40.

Kats, D. et R. L. Kahn, The Social Psychology of Organizations. New York, John Wiley \& Sons, 1966.

Kopec, J. A., The Communication Audit, Public Relations Journal, mai 1982, pp. 24-27.

Machin, J. L. J. et C. H. S. Tai, Senior Managers Audit Their Own Communications, Journal of Entreprise Management, vol. 2, $\mathrm{n}^{\circ}$ 1,1979, pp. 75-85.

Mackenzie, K. D., Organizational Design : The Organizational Audit and Analysis Technology. Norwood, N.J., Ablex Publishing Corporation, 1986.

Mikol, A., Dans la jungle des Audits, Annales des Mines : Gérer et Comprendre, décembre 1991, pp. 4-10.

Morgan, G., Images de l'organisation. Québec, Les Presses de l'Université Laval/Éditions Eska, 1989.

N.S.P.E., How to Improve Engineering-Management Communications. Washington, D.C., National Society of Professional Engineers, 1952.

Odiorne, G. An Application of the Communication Audi, Personnel Psychology, vol. 7, 1954, pp. 235-243.

Pemartin, D., Mythes d'entreprise et communication interne. Paris, Les Éditions d'Organisation, 1990.

Rigny, A. J., Diagnostic organisationnel. Montréal, Les Éditions Agence d'Arc, 1982.

Sincoff, M. Z et R. S. Goyer, Communication Audit Critique : The Researcher's Perspective, Journal of Business Communication, vol. 15,1977, pp. 57 à 63.

Strenski, J. B., The Communication Audit : Primery P. R. Measurement Tool, Public Relations Quarterly, vol. $23, \mathrm{n}^{\circ} 4,1978$, pp. 17-18.

Strenski, J. B., The Communication Audit-Basic to Business Development, Public Relations Quarterly, vol. $29, \mathrm{n}^{\circ} 1,1984$, pp. 14-17.

Valentine, J., Audit of Administrator Communication, Peabody Journal of Education, vol. 59, 1981, pp. 1-10.

Vardaman, G. T. et C. C. Vardaman, La communication au service du contrôle de gestion. Les systèmes de diagnostic et de conception des organisations. Paris, Éditions Chiron, 1974.

Washbrook, H., The Board and Management. London, Business Books, 1978.

Wirtz, J. R., The Communication Audit - Your Road Map to Success,Journal of Organizational Communication, vol. 10, $\mathrm{n}^{\circ}$ 2, 1981, pp. 15-17. 


\section{RÉSUMÉS}

L'auteur fait état de l'origine et des caractéristiques des pratiques d'audit. Par la suite, il traite des problèmes relatifs aux audits de communication. Il fait une synthèse des différentes facettes de la fonction d'auditeur et analyse les facteurs qui peuvent influencer leur pratique.

The author established the origin and characteristics of auditing. Subsequently, he (she) deals with the problems associated with communications audits. He (she) brings together the various components of an auditor's task and examines the factors influencing their usage.

\section{AUTEUR}

\section{GILLES WILLETT}

Professeur et chercheur au Département d'information et de communication de l'Université Laval et administrateur à la Faculté des arts. Ses travaux portent sur la communication dans les organisations, le phénomène de la télécommunication et ses technologies, les théories de la communication et le droit de communiquer. Il a donné plusieurs communications scientifiques dans le cadre de congrès nationaux et internationaux. 